\title{
Ways to increase the evaporation surface area of liquid droplets: an experimental study
}

\author{
Dmitrii V. Antonov', Genii V. Kuznetsov', Maxim V. Piskunov', Pavel A. Strizhak', Nikita E. Shlegel ${ }^{1}$ \\ ${ }^{1}$ National Research Tomsk Polytechnic University \\ 30 Lenin Avenue, Tomsk 634050, Russia \\ pavelspa@tpu.ru
}

\begin{abstract}
This study presents the experimental findings on the outcomes of homogeneous water droplets collision in an aerosol cloud and heterogeneous water droplet breakup in the counter gas flow, e.g., airflow. The temperature of such a flow varied from $20^{\circ} \mathrm{C}$ to 500 ${ }^{\circ} \mathrm{C}$. The initial size, namely radius, of the heterogeneous droplets was $1-1.5 \mathrm{~mm}$. The outcomes of collision and breakup of droplets were recognized by high-speed cameras and tracking algorithms of Tema Automotive and Mathematica software. For the first time, we offered the criterion characterizing a change in the liquid surface area due to the enhancement of droplets collision in a gas environment and heterogeneous droplet breakup.
\end{abstract}

Keywords: Homogeneous water droplet, heterogeneous water droplet, evaporation surface area, breakup, micro-explosion, puffing.

\section{Introduction}

Nowadays, the global scientific community focuses on a utilization of multiphase and multicomponent gas-vapordroplet media in a large group of technologies [1,2]. This fact is primarily due to that such an approach promotes higher ecological, energy, technical and economic indicators of equipment operation. For instance, there are technologies providing for a growth of the mentioned indicators by $20-60 \%$ when using the multiphase and multicomponent gas-vapor-droplet media [3]. Among of these technologies are thermal cleaning of water and other liquids from impurities; heat-power equipment with the enhanced evaporation and condensation; heat carriers based on flue gases, vapors, and droplets of water; ignition of composite fuels excluding the nozzle clogging and the flame extinction in combustion chambers; fire extinguishing by the gas-vapor-droplet flows. However, the main problem of multiphase and multicomponent gas-vapordroplet media for wide using is a complexity in predicting their structure and composition [3].

One of the promising methods for the considerable enhancement of phase transition in multiphase and multicomponent gas-vapor-droplet media is an increase in evaporation surface area due to the fine liquid spraying and the explosive breakup of droplets. Actually, the explosive breakup is a secondary atomization of liquids directly within the intense heat-exchange zone [4]. Researchers across the globe attempt to develop technologies based on this phenomenon. However, the study of an attainment of conditions for the explosive breakup of droplets with a formation of the fuel aerosol is the most attractive (from the viewpoint of the suitable consumption of power and time) one [5-7].

The purpose of the study is to determine, based on experiments, the outcomes of homogeneous water droplets collision and heterogeneous water droplet breakup at different heating temperatures.

\section{Experimental methods and setup}

Fig. 1 depicts the schemes of the experimental setups for carrying out experiments on spraying an aerosol $(a)$ and on heterogeneous droplet breakup $(b)$. 


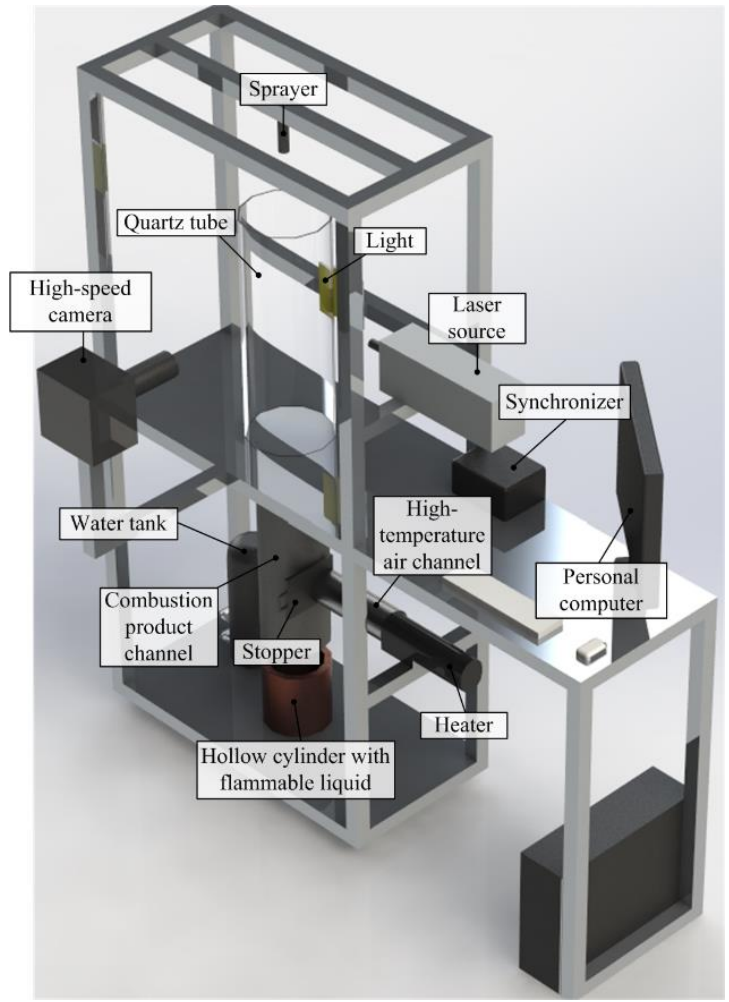

$a$

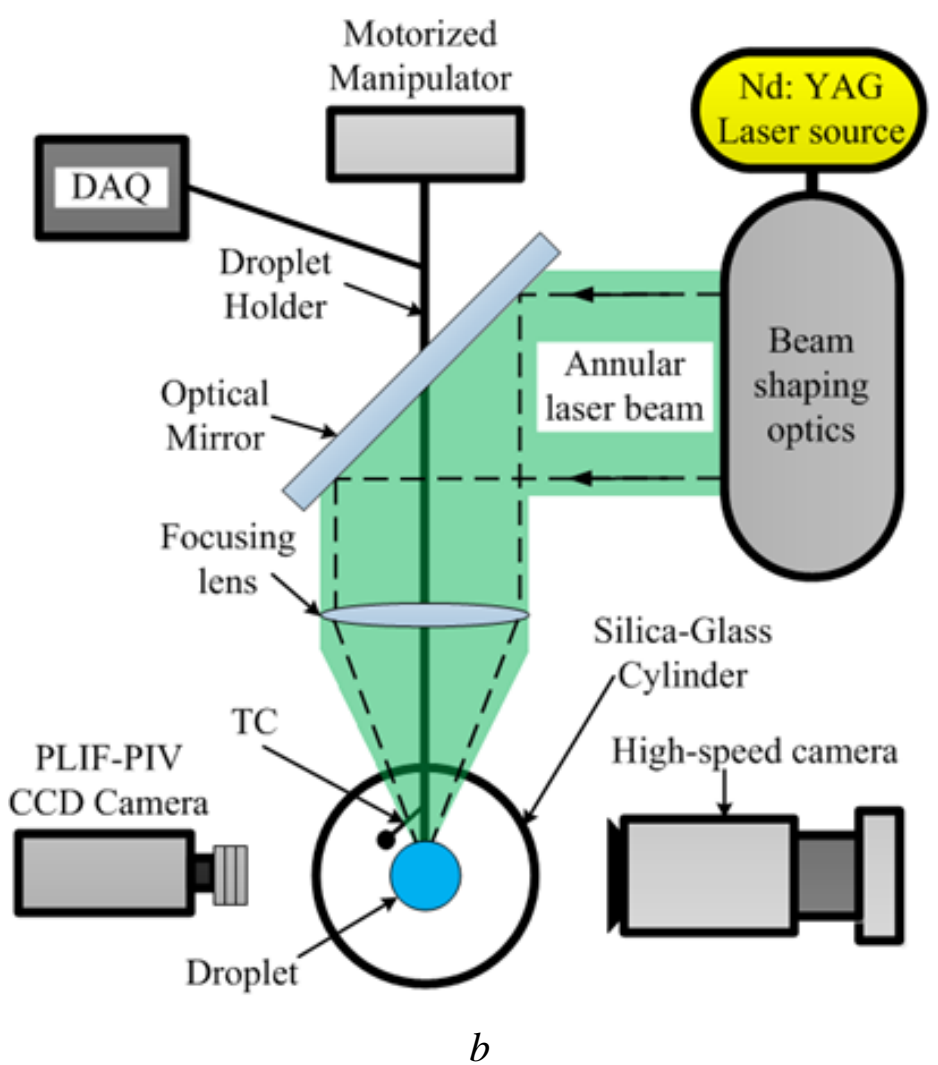

$b$

Fig. 1: Schemes of the experimental setups: $a$-experiments on spraying an aerosol; $b$-experiments on heterogeneous droplet breakup.

\subsection{Collisions of aerosol droplets}

Fig. 1 a presents a scheme of the setup equipped with gas (air) flow blower and heater, quartz cylinder with a gas flow produced, aerosol generator, software and hardware system of tracking. We additionally performed experiments with a generating a flow of combustion products of kerosene when varying their temperature (around 800-850 ${ }^{\circ} \mathrm{C}$ ) and velocity (from $0 \mathrm{~m} / \mathrm{s}$ to $10 \mathrm{~m} / \mathrm{s}$ ). To do that, we applied a burner (hollow cylinder with flammable liquid) instead of gas flow blower and heater. The gas temperature was measured by three fast-response type-K thermocouples with a measurement range of $-50-1200{ }^{\circ} \mathrm{C}$, an error of $\pm 1.5^{\circ} \mathrm{C}$, and a junction thickness of $0.3 \mathrm{~mm}$. The National Instruments 9213 module collected the thermocouples measurement data. The relative concentration of $0.001-0.002 \mathrm{~m}^{3}$ water droplets per one $\mathrm{m}^{3}$ gas was maintained on the same level during all the experiments to correspond the real spray technologies.

The experimental study of the outcomes of water droplets collision was performed under the one-second pulse injection of polydisperse water aerosol with a size (radius) of droplets from $0.05 \mathrm{~mm}$ to $1 \mathrm{~mm}$ and velocity $\left(U_{\mathrm{d}}\right)$ from $0 \mathrm{~m} / \mathrm{s}$ to 10 $\mathrm{m} / \mathrm{s}$. To measure the gas flow velocity $\left(U_{\mathrm{a}}\right)$, we applied the optical technique Particle Image Velocimetry (PIV) [8].

To keep track of the moving droplets, we used the corresponding algorithms of the Tema Automotive software [9].

\subsection{Breakup and explosion of droplets}

Fig. $1 b$ illustrates the scheme of recording the outcomes of the heterogeneous droplet breakup at the convective heating. A heating system consists of the Leister CH 6060 hot-air blower (air velocity $0.5-5 \mathrm{~m} / \mathrm{s}$ ) and the Leister LE 5000 HT air heater (temperature range $20-1000{ }^{\circ} \mathrm{C}$ ) generating the gas flow with suitable velocity $U_{\mathrm{a}}$ and temperature $T_{\mathrm{a}}$. The flow is produced inside the hollow transparent cylinder made from silica glass with an inner diameter of $0.1 \mathrm{~m}$ and a wall thickness of $2 \mathrm{~mm}$. This cylinder has three holes with the diameter of $10 \mathrm{~mm}$ for laser lighting of droplets, for their introducing in the gas flow, and for recording the processes under study. A gas temperature inside the cylinder was measured by the system 
consisting of the National Instruments 9213 module collecting the measurement data and two fast response type-K thermocouples with a measurement range of $0-1200{ }^{\circ} \mathrm{C}$, an accuracy of $\pm 1{ }^{\circ} \mathrm{C}$, and a time lag of $0.1 \mathrm{~s}$.

The gas flow velocity $U_{\text {a }}$ was measured by the optical technique PIV [8]. The values $U_{\text {a }}$ were determined before the main experiment, i.e. before introducing a droplet in the cylinder. An error in determining $U_{\mathrm{a}}$ was less than $2 \%$. Since within within the scheme with the convective heating the gas flow had sufficiently high temperatures $T_{\mathrm{a}}$, then the values $U_{\mathrm{a}}$ were were possible to measure only by the contactless optical technique.

The two-component droplet under study is placed at a steel holder with a diameter of $0.62 \mathrm{~mm}$. A motorized manipulator moves this holder in the gas flow. The high-speed camera allowed recording the micro-explosion and puffing processes of the two-component droplets. Note that in the experimental study we also measured a temperature inside droplets by using the optical technique Planar Laser-Induced Fluorescence; see more details about the procedure of such measurements in research [8].

\subsection{The registered parameters and measurement errors}

The maximum random errors were no more than $20 \mathrm{~K}$ when measuring $T_{\mathrm{a}}$, and no more than $3 \mathrm{~K}$ when measuring $T_{\mathrm{d}}$. Approximation expressions described by exponential and power functions were obtained for the drop decay times $(\tau)$, as well as for the warm-up times of the drop and its evaporation while maintaining integrity $\left(\tau_{\mathrm{h}}\right)$. In addition, for each time dependence, confidence intervals were determined to illustrate the variation of experimental values (measurement errors $<4 \%$ ). For the $S / S_{0}$ area ratio, the diagrams have been obtained showing the confidence intervals.

\section{Results and Discussion}

The bar graphs illustrating the liquid surface area growth $\left(S / S_{0}\right)$ during the droplets breakup in a sprayed flow due to collisions (Fig. 2a) and micro-explosion and puffing processes (Fig. 2b) characterize the main experimental findings on the outcomes of a collision of the homogeneous droplets and a breakup of the heterogeneous droplets.

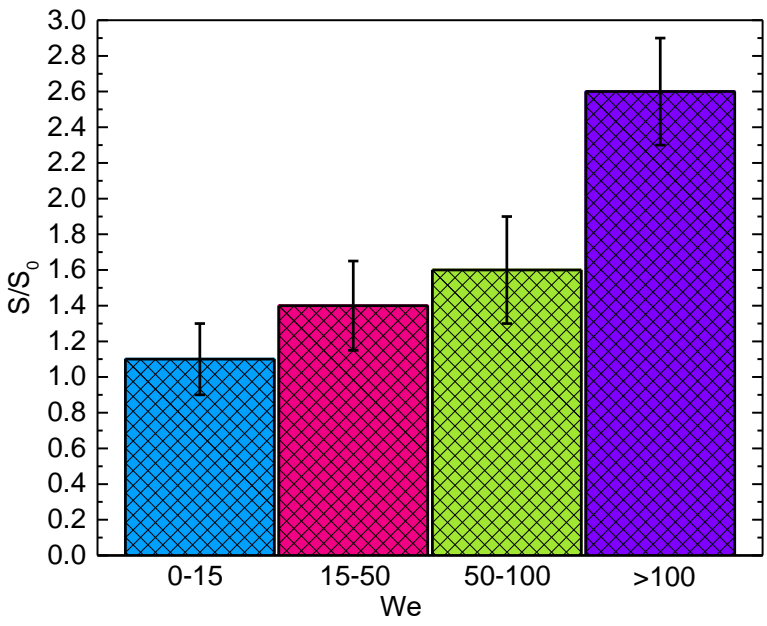

$a$

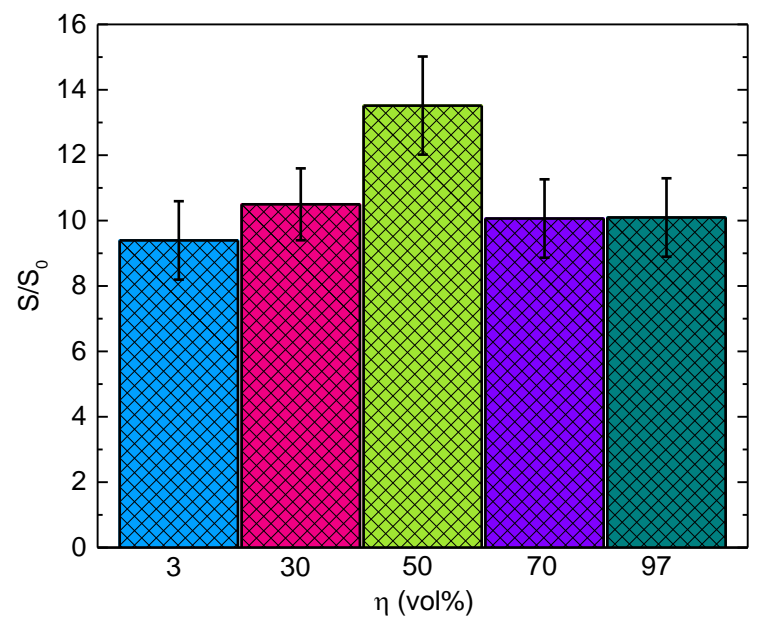

$b$

Fig. 2: Bar graphs illustrating the liquid surface area growth: a - in the case of the droplets breakup due to collisions (convective heating, $T_{\mathrm{a}}=350{ }^{\circ} \mathrm{C}$, water droplets, We - Weber number); $b$ - in the case of the droplets breakup due to micro-explosion and puffing (convective heating, $T_{\mathrm{a}}=350{ }^{\circ} \mathrm{C}$, heterogeneous water-oil droplets, $\eta$ - turbine oil concentration).

Fig. 2 demonstrates a difference between ratios $S / S_{0}$ for the two mentioned mechanisms of increasing the evaporation surface area. The values $S / S_{0}$ presented in Fig. $2 a$ were calculated as mean ones for all the collisions recorded; the values $S / S_{0}$ in Fig. $2 b$ - as mean ones for all the recorded breakups of the heterogeneous droplets. The total evaporation surface area 
in the experiments with the sprayed flow altered by 1.5-2 times, i.e. insignificantly because in the experiments with the heterogeneous droplets, the evaporation surface area increased 9-14-fold.

Each of the mechanisms examined taking into account thermal (overheat), aerodynamic (acceleration), and contact (collision) factors effectively promotes the growth in the $S / S_{0}$ ratio. This result is important for developing heat mass transfer technologies based on the phase transition enhancement through the secondary atomization of droplets directly within a chamber. The secondary atomization is possible to implement by the combination of the three factors under discussion. In case, the energy expenditures for accelerating droplets, their colliding or preparing the heterogeneous liquids should be considered.

\section{Conclusion}

The two main mechanisms of increasing in evaporation surface area were revealed in the experimental study. These are breakup due to the droplets collision in the sprayed flow as well as micro-explosion and puffing of the heterogeneous droplets. In the case of the heterogeneous droplet breakup, the evaporation surface area increases 9-14-fold; in the case of the homogeneous droplet breakup in the sprayed flow -1.5-2-fold, i.e. the breakup due to collisions is less effective. Based on the experimental findings, it is possible to predict the total evaporation surface area of a liquid as one of the most important characteristics in modern gas-vapor-droplet systems.

\section{Acknowledgements}

This study was financially supported by the Russian Science Foundation (project 18-71-10002).

\section{References}

[1] S. Paruchuri, M. P. Brenner, "Splitting of a jet," Phys. Rev. Lett., vol. 98.

[2] A.Y. Varaksin, "Fluid dynamics and thermal physics of two-phase flows: Problems and achievements," High Temp., vol. 51, pp. 377-407, 2013.

[3] O. V. Vysokomornaya, G. V. Kuznetsov, P. A. Strizhak, "Evaporation and transformation of droplets and large massifs of a liquid during motion through high-temperature gases," Novosibirsk, SB RAS, pp. 302, 2016.

[4] M. V. Piskunov, P. A. Strizhak, "Using Planar Laser Induced Fluorescence to explain the mechanism of heterogeneous water droplet boiling and explosive breakup,” Exp. Therm. Fluid Sci., vol. 91, pp. 103-116, 2018.

[5] Y. Suzuki, T. Harada, H. Watanabe, M. Shoji, Y. Matsushita, H. Aoki, T. Miura, "Visualization of aggregation process of dispersed water droplets and the effect of aggregation on secondary atomization of emulsified fuel droplets," in Proc. Comb. Inst., vol. 33, pp. 2063-2070, 2011.

[6] D. Tarlet, C. Josset, J. Bellettre, "Comparison between unique and coalesced water drops in micro-explosions scanned by differential calorimetry," Int. J. Heat Mass Transfer, vol. 95, pp. 689-692, 2016.

[7] Z. Yin, P. Nau, W. Meier, "Responses of combustor surface temperature to flame shape transitions in a turbulent bistable swirl flame," Exp. Therm. Fluid Sci., vol. 82, pp. 50-57, 2017.

[8] G. V. Kuznetsov, M. V. Piskunov, R. S. Volkov, P. A. Strizhak, "Unsteady temperature fields of evaporating water droplets exposed to conductive, convective and radiative heating," Appl. Therm. Eng., vol. 131, pp. 340-355, 2018.

[9] J. E. Sprittles, Y. D. Shikhmurzaev, "Coalescence of liquid drops: Different models versus experiment," Phys. Fluids, vol. 24, 2012. 